\title{
Sonographic comparison of transcutaneous stimulation versus percutaneus stimulation of the tibialis anterior: a pilot study
}

\author{
Sánchez Lorenzo M. ${ }^{1}$ Seoane Pardo R. ${ }^{1}$ Mira Llopis M. ${ }^{2}$ lannone Lado S. ${ }^{2}$ \\ ${ }^{1}$ Tambre Fisioterapia, Sigüeiro, A Coruña, Spain \\ 2 Fisioterapia A Xunqueira, Cee, A Coruña, Spain \\ Rev Fisioter Invasiva 2019;2:93.
}

\begin{abstract}
Background and Aims Previous studies have evaluated electrostimulation of the tibialis anterior muscle via ultrasound. However, to the best of our knowledge, to date, no study has compared percutaneous stimulation compared to transcutaneous stimulation. The aim of this study was to analyze and compare the influence of percutaneous stimulation versus transcutaneous stimulation on the angle and muscle width of the proximal motor point of the tibialis anterior among healthy individuals using ultrasound.

Material and Methods A longitudinal prospective study. The study variables were muscle thickness and pennation angle, measured using ultrasound. A sample of 4 healthy individuals with a mean age of 35.25 years $( \pm 2.17)$, mean height of $1.70 \mathrm{~m}$ $( \pm 0.03)$ and weight of $67.35 \mathrm{~kg}( \pm 6.32)$, participated in this study. Stimulation was performed on the tibialis anterior of the dominant leg of each individual $(n=4)$. The subjects were seated in a vertical position. For position 1, the knee of the dominant leg remained completely extended and the ankle was fixed in a neutral position with an orthosis comprised of Velcro straps which immobilized the ankle and forefoot joints. For position 2, the knee remained flexed 90 degrees with the foot fixed in the orthosis and supported on the floor. The proximal motor point of the tibialis anterior muscle was located. A biphasic symmetric pulse current was used with the maximum tolerated intensity. Transcutaneous stimulation was performed via a small circular electrode, and for percutaneous stimulation a filiform acupuncture needle was used. To capture the ultrasound images, the probe was placed on a system with an articulated mechanical arm and a clamp that enabled the possibility of adjusting the height and/or angle and the position marked on the skin. Normality was contrasted using the Shapiro-Wilk test and sphericity was tested using the Mauchly's test. Analysis of variance was performed (ANOVA) for repeated measures.

Results The comparison of both techniques in position 1 did not show significant differences between the transcutaneous technique versus the percutaneous technique neither for the angle $(F=2.07$; $p$-valor $=0.18)$, nor for the width $(F=0.28$; $p$ value $=0.60$ ). In the case of position 2 , significant differences were not found between

\section{Keywords}

- ultrasonography

- electric stimulation

- tibialis anterior muscle the transcutaneous technique versus the percutaneous technique, neither for the angle $(F=0.28$; $p$-value $=0.606)$ nor for the weight $(F=0.11$; $p$-value $=0.75)$.

Conclusions The comparison of transcutaneous stimulation versus percutaneous stimulation in the proximal motor point of the tibialis anterior does not seem to show statistically significant differences for muscle width nor pennation angle.
\end{abstract}

DOI https://doi.org/

$10.1055 / \mathrm{s}-0039-3401872$

ISSN 2386-4591.
Copyright (c) 2019 by Thieme Revinter

Publicações Ltda, Rio de Janeiro, Brazil
License terms

(ㅇ)(1) $\Theta$ 